\title{
Proceedings
}

\section{Giving New Uses to Glucose Meters: Detection of Prostate Cancer ${ }^{\dagger}$}

\author{
Clara Abardía-Serrano ${ }^{1,2, *}$, Rebeca Miranda-Castro ${ }^{1,2}$, Noemí de-los-Santos-Álvarez ${ }^{1,2}$ and \\ María Jesús Lobo-Castañón 1,2 \\ 1 Departamento de Química Física y Analítica, Universidad de Oviedo. Av. Julián Clavería 8, 33006 Oviedo, \\ Spain; mirandarebeca@uniovi.es (R.M.-C.); santosnoemi@uniovi.es (N.d.-1.-S.-Á.); \\ mjlc@uniovi.es (M.J.L.-C.) \\ 2 Instituto de Investigación Sanitaria del Principado de Asturias. Av. de Roma, 33011 Oviedo, Spain \\ * Correspondence: abardiaclara@uniovi.es \\ + Presented at the 1st International Electronic Conference on Biosensors, 2-17 November 2020; Available \\ online: https://iecb2020.sciforum.net/.
}

Received: date; Accepted: date; Published: date

\begin{abstract}
A sandwich genoassay for the detection of PCA3, a nucleic acid biomarker overexpressed in the urine of prostate cancer patients, has been developed by using the enzyme alkaline phosphatase (ALP) as a tracer of the hybrid generated onto the surface of magnetic particles. ALP converts D-glucose-1-phosphate into D-glucose, which is quantified with a personal glucose meter. The resulting methodology allows the reliable detection of PCA3 at low picomolar levels, thus fostering the massive screening of prostate cancer.
\end{abstract}

Keywords: nucleic acid detection; genoassay; prostate cancer; personal glucose meter

\section{Introduction}

Liquid biopsy has emerged as a potential supplement of traditional biopsy for early cancer diagnosis. It consists in monitoring the level of tumor biomarkers present in appropriate body fluids. Consequently, the development of non-invasive, easy-to-use and inexpensive methods for the detection of biomarkers is highly demanded for improving diagnosis and minimizing the mortality of cancer. In this context, electrochemical platforms have demonstrated great potential due to their high sensitivity, low cost, fast response as well as compatibility with point-of-care testing (POCT), what contributes to decentralize the analyses and favors the implementation of screening studies. However, putting into the market a new sensing platform for detecting a biomarker of interest is a long-distance race. To accelerate the process, a modern strategy consists in adapting or repurposing already approved and commercialized technology to the detection of targets different from those originally intended. In this sense, the personal glucose meter (PGM), the most used (electro)analytical method worldwide that satisfies all the requirements demanded from POCT devices, has been employed for the detection of many other targets [1]. Following this trend, we have recently reported on the conversion of the PGM into an electrochemical transducer of hybridization events for monitoring a nucleic acid approved by the US Food and Drug Administration (FDA) as a urinary biomarker for prostate cancer diagnosis, the so-called PCA3 [2]. Herein, we discuss different approaches evaluated and highlight the main achievements. It could be a valuable information for other researchers interested in exploiting the PGM beyond glucose control. 


\section{Materials and Methods}

\subsection{Reagents}

The DNA sequences used in this work are: target PCA3(ТPCA3): AAGCAAAATACTTGCATTAGGTCTCAGCTGGGGCTGTGCATCAGGCGGTTTGAGAAATATT CAATTCTCAGCAGAAGCCAGAATT; biotinylated capture probe (b-CP): Biotin-(T)6-AATTCTG GCTTCTGCTGAGAAT; 6FAM-signaling probe (SP-6FAM): AGACCTAATGCAAGT-6FAM; auxiliary probe (AuxP): ACCGCCTGATGCACAG; interferent PSA: GGT CTTCCTTTGGCATGGGATGGGGATGAAGTAAGGAGAGGGACTGGACCCCCTGGAAGCTGAT T; all of them were synthesized and HPLC purified by Metabion (Planegg, Germany).

\subsection{Instrumentation}

GlucoMen ${ }^{\circledR}$ AERO 2K personal glucose meter was employed for measuring the glucose concentration. Throughout the magnetogenoassay, a Dynabeads ${ }^{\text {TM }}$ MX 12-tube Mixing Wheel was used as a form of agitation, and for the stages in which thermal control was necessary, a ThermoMixer Comfort (Eppendorf, Spain) was used. Spectrophotometric measurements required in Trinder method implementation were carried out with a GENESYSTM $10 S$ UV-Vis spectrophotometer, with plastic cuvettes of $1 \mathrm{~cm}$ optical path length.

\subsection{Sensing Layer Preparation onto $M B$}

Formation of the sensing layer begins with the modification of the magnetic beads (MBs) with the biotinylated PCA3 capture probe, through the streptavidin-biotin binding. To block the streptavidin molecules that remain free on the surface of the particles, a blocking stage is subsequently carried out with biotin. Finally, after washing to remove the biotin in excess, MBs were redispersed in the appropriate buffer and stored at $4{ }^{\circ} \mathrm{C}$ until use.

\subsection{Hybridization-based Assay onto $M B$}

The sandwich assay that allows the detection of PCA3 starts with a homogeneous hybridization stage between variable analyte concentrations and excess of signaling and auxiliary probes, which are subjected to thermal shock $\left(98{ }^{\circ} \mathrm{C}\right.$ for $5 \mathrm{~min}$ and cooling to RT). The following stage is a heterogeneous hybridization between the ternary duplex performed in solution and the sensing layer during $2 \mathrm{~h}$ at RT. Previously, BSA at $5 \%$ is added to promote the on-surface hybridization. Once the unbound DNA was removed, the magnetic beads are brought into contact with the enzyme conjugate ( $2 \mathrm{U} / \mathrm{mL}$ antifluorescein Fab fragment-6-carboxyfluorescein-Alkaline phosphatase conjugate, antiFALP) in the presence of casein to minimize unspecific interactions, during $30 \mathrm{~min}$ at RT. The resulting modified magnetic beads were thoroughly washed and resuspended in the hydrolysis buffer $(0.1 \mathrm{M}$ diethanolamine $\mathrm{pH}$ 9.8, $1 \mathrm{mM} \mathrm{MgCl}$ ), which contains glucose-1-phopshate, the precursor of Dglucose. Enzymatic hydrolysis reaction is carried out at $37^{\circ} \mathrm{C}, 1300 \mathrm{rpm}$, for $2 \mathrm{~h}$. Finally, the supernatant containing the glucose generated was recovered and neutralized with $2 \mathrm{M} \mathrm{HCl}$, in order to perform the measurement with the personal glucose meter (PMG).

\section{Results and Discussion}

We have designed and developed a method for the detection of a nucleic acid biomarker for prostate cancer diagnosis called prostate cancer antigen 3 (PCA3), by combining magnetic beads $(\mathrm{MBs})$ as the platform for a sandwich-type genoassay with a personal glucose meter (PGM) as the signal transducer.

In the proposed sandwich-hybridization assay a target sequence specific of PCA3 is hybridized with three probes: the capture probe, which is tethered to magnetic beads by means of streptavidinbiotin binding for sensing layer construction (Figure 1a), the signaling probe including a suitable tag for subsequent attachment of the tracer enzyme, and an auxiliary probe). This bioassay is conducted 
in two steps. First, solution-phase hybridization between the target and the auxiliary and signaling probes is performed. The resulting ternary duplex is then captured onto the sensing layer through solid-phase hybridization (Figure 1b).

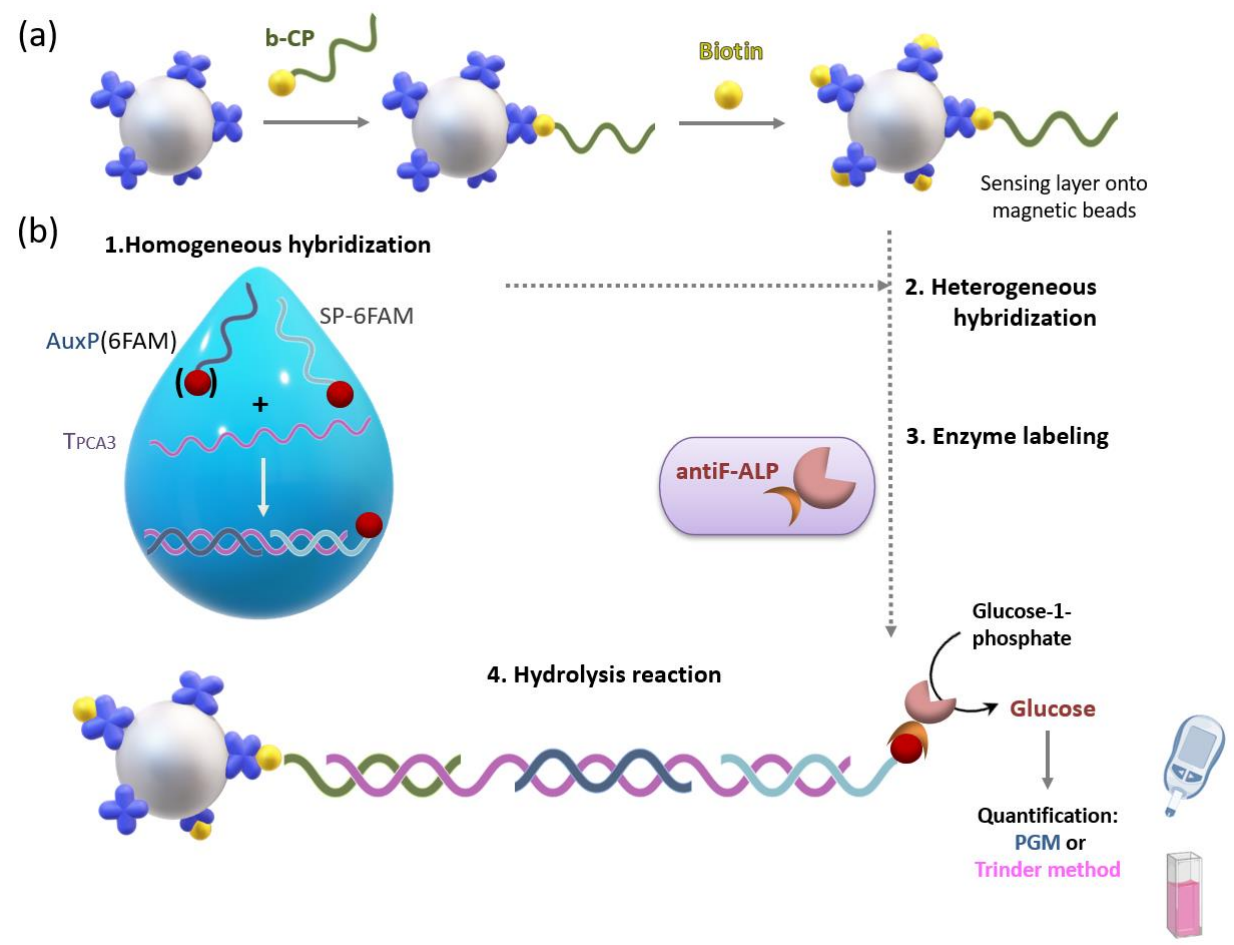

Figure 1. Scheme of the sensing layer construction (a), and the sandwich genoassay for the detection of PCA3 (b).

In order to link the amount of PCA3 to the generation of glucose for subsequent quantification with the PGM, several enzymes capable of catalyzing the conversion of a glucose precursor into glucose were evaluated as a tracer of the sandwich genoassay. In each case, the concentration of the glucose precursor enzymatic substrate and the enzymatic reaction time were optimized. Since the PGM possesses a narrow response range (20-600 mg/dL), the optimization of these parameters would result cumbersome and require a high consumption of glucose test strips. For this reason, these studies were conducted by measuring the produced glucose concentration with a fixed-time enzymatic colorimetric method, the so-called Trinder method [3].

Once the glucose generation conditions were established, the magnetic beads modified with the sensing layer were challenged to increasing concentrations of PCA3. The best analytical performance was accomplished when using alkaline phosphatase (ALP) as the enzyme generating glucose from D-glucose-1-phosphate. ALP was attached to the hybrid formed onto the magnetic beads in the presence of PCA3 by either biotin-streptavidin (biot-strep) or antifluorescein Fab fragment-6carboxyfluorescein (antiF-6-FAM) binding, thus requiring biotin-tagged or a 6-FAM-tagged signaling probes, respectively. The monovalent affinity reaction of antiF-6-FAM turned out to be more convenient since precludes the labeling of several hybrids with the same enzyme molecule, as was the case for the biotin-streptavidin interaction due to the multivalence of the protein containing four biotin binding sites.

Under these conditions, an increase in the glucose concentration generated by ALP was observed, which is directly proportional to the amount of PCA3 in the range from $10 \mathrm{pM}$ to $100 \mathrm{pM}$, both with PGM-based and spectrophotometric methods. However, the sensitivity of the PGM-based method was $(2.8 \pm 0.1) \mathrm{mg}$ glucose $\mathrm{dL}^{-1} \mathrm{pM}^{-1}$, that is 1.6 times higher than that of the Trinder-based method, showing the suitability of the POCT device. 
In order to detect lower PCA3 concentrations, the auxiliary probe designed to help in target hybridization was also labeled with 6FAM, doubling the antiF-ALP binding sites per hybrid formed. This strategy led to an improved sensitivity $\left(3.3 \pm 0.2 \mathrm{mg}\right.$ glucose $\left.\mathrm{dL}^{-1} \mathrm{pM}^{-1}\right)$, pushing the experimental limit of quantification down to $5 \mathrm{pM}$. The method selectivity was also evaluated by using a DNA homolog of PSA mRNA, which is present in men urine. A concentration of the interference equivalent to the upper limit of the PCA3 response range, that is $100 \mathrm{pM}$, gave rise to a PGM signal below $20 \mathrm{mg}$ glucose/ $\mathrm{dL}$, which is indicative of excellent selectivity.

Funding: This research was funded by the Spanish Ministerio de Ciencia y Universidades, grant number RTI2018-095756-B-I00, and Principado de Asturias Government, grant number IDI/2018/000217, co-financed by FEDER funds.

Acknowledgments: We thank Carmen Rodríguez from A. Menarini Diagnostics (Barcelona, Spain) for her kind donation of the personal glucose meter.

Conflicts of Interest: The authors declare no conflict of interest.

\section{References}

1. Lisi, F.; Peterson, J.R.; Gooding, J.J. The application of personal glucose meters as universal point-of-care diagnostic tools. Biosens. Bioelectron. 2020, 148, 111835, doi:10.1016/j.bios.2019.111835.

2. Abardía-Serrano, C.; Miranda-Castro, R.; de-los-Santos-Álvarez, N.; Lobo-Castañón, M.J. New uses for the personal glucose meter: detection of nucleic acid biomarkers for prostate cancer screening. Sensors 2020, 20, 5514, doi:10.3390/s20195514.

3. Barham, D.; Trinder, P. An improved color reagent for the determination of blood glucose by the oxidase system. Analyst 1972, 97, 142-145, doi:10.1039/AN9729700142.

(C) 2020 by the authors. Submitted for possible open access publication under the terms and conditions of the Creative Commons Attribution (CC BY) license (http://creativecommons.org/licenses/by/4.0/). 